\title{
Does food insulin index in the context of mixed meals affect postprandial metabolic responses and appetite in obese adolescents with insulin resistance? A randomised cross-over trial
}

\author{
Z. Caferoglu'*, N. Hatipoglu² and H. Gokmen $\mathrm{Ozel}^{3}$ \\ ${ }^{1}$ Department of Nutrition and Dietetics, Faculty of Health Sciences, Erciyes University, Kayseri, Turkey \\ ${ }^{2}$ Department of Paediatric Endocrinology, Faculty of Medicine, Erciyes University, Kayseri, Turkey \\ ${ }^{3}$ Department of Nutrition and Dietetics, Faculty of Health Sciences, Hacettepe University, Ankara, Turkey \\ (Submitted 18 January 2019 - Final revision received 7 May 2019 - Accepted 30 May 2019 - First published online 23 September 2019)
}

\section{Abstract}

The food insulin index (II) is a novel classification to rank foods based on their physiological insulin demand relative to an isoenergetic reference food and may be a valid predictor of postprandial insulin responses and appetite. The present study aimed to compare the postprandial metabolic responses and appetite sensations to two macronutrient- and glycaemic index-matched meals with either high or low II in obese adolescents with insulin resistance (IR). A randomised, single-blind and cross-over trial included fifteen obese adolescents aged 12-18 years with IR. All participants were provided with two different breakfasts: low glycaemic index, low insulin index (LGI-LII) and low glycaemic index, high insulin index (LGI-HII), with a 1-week washout period between meals. At time 0 (just before breakfast), 15, 30, 45, 60, 90, 120, 180 and $240 \mathrm{~min}$ after the meal, serum glucose, insulin and C-peptide levels and appetite scores were measured. At the end of $4 \mathrm{~h}$, participants were served ad libitum lunch. Early (0-30 $\mathrm{min})$, late ( $45-240 \mathrm{~min})$ and total (0-240 min) postprandial insulin responses were lowered by $56 \cdot 1,34 \cdot 6$ and $35.6 \%$ after the LGI-LII meal $v$. LGI-HII meal $(P<0.05)$. The feeling of hunger was also decreased by 25.8 and $27.5 \%$ after the LGI-LII meal $v$. LGI-HII meal during the late and total responses $(P<0.05)$. The calculation II of meals or diets may be a useful dietary approach to reduce postprandial hyperinsulinaemia and the perceived hunger in obese adolescents with IR.

Key words: Food insulin index: Glycaemic index: Obesity: Insulin resistance: Adolescents

Obesity is one of the major public health problems in the world, and adolescence is a critical period in the development of obesity $^{(1)}$. Treatment of obesity in the adolescence period is important in terms of both protecting the health and preventing the diseases in the adulthood. However, little is known about the optimal dietary approach for weight loss in obese adolescents, particularly for those at risk of developing type 2 diabetes. The traditional treatment approach focuses on reducing energy intake by decreasing fat and increasing carbohydrates; however, this option may not be appropriate to treat obese adolescents with insulin resistance (IR). It is speculated that this diet may lead to higher levels of postprandial glycaemia and/or insulinaemia and may increase IR, with a potential role in the development of type 2 diabetes $^{(2)}$.

Recently, the role of carbohydrate quality as assessed by glycaemic index (GI) in obesity management, especially for individuals with a compensatory increased insulin secretion, has received considerable attention. A diet with a low GI is hypothesised to stimulate increased satiety and reduce voluntary energy intake due to the slower blood glucose and insulin response following consumption ${ }^{(1,2)}$. Although carbohydrates are the major stimulus for insulin secretion, they are not the only one. Dietary proteins and fats also elicit a significant insulin response ${ }^{(2,3)}$. The new concept of the food insulin index (II), which directly quantifies the postprandial insulin response to a test food in comparison with an isoenergetic portion of a reference food (either glucose or white bread), has been suggested to be more suitable than GI in evaluating conditions related to insulin exposure, like obesity ${ }^{(4)}$.

Nevertheless, few studies have been conducted to elucidate the role of II in obesity. In a study investigating the effect of dietary GI and II on body composition, a higher dietary II during puberty was associated with a higher percentage of body fat in young adulthood, although dietary GI during puberty was

Abbreviations: GI, glycaemic index; iAUC, incremental AUC; II, insulin index; IR, insulin resistance; LGI-HII, low glycaemic index and high insulin index; LGI-LII, low glycaemic index and low insulin index.

* Corresponding author: Z. Caferoglu, fax +90 352437 9281, email zeynepcaferoglu@gmail.com; zcaferoglu@erciyes.edu.tr 
not related to body composition in young adulthood ${ }^{(5)}$. Furthermore, a recent study has shown that diets with low insulin demand may reduce energy intake and may hence assist with weight loss in obese adolescents with $\mathrm{IR}^{(2)}$. On the other hand, single-food studies found the II as a better predictor of observed insulin responses than the carbohydrate content or GI. However, it has been yet unknown whether the II in the context of realistic mixed meals can affect the postprandial glucose and insulin responses in obese adolescents with IR. The effect of II on short-term hunger, satiety or voluntary energy intake has not been studied. Therefore, the present study aimed to determine whether II could affect postprandial metabolic responses and appetite following consumption of test meals with similar macronutrient and GI, but different II amounts in obese adolescents with IR.

\section{Methods \\ Participants}

Twenty-two obese adolescents who consecutively attended the outpatient clinic of the Paediatric Endocrinology at the Erciyes University Children's Hospital were included on the basis of the following criteria: aged between 12 and 18 years, newly physician-diagnosed obesity and IR, and absence of the previous obesity and/or IR treatment including diet therapy. All participants were examined by the paediatric endocrinologist before the study. Obesity was defined according to the BMI (weight in $\mathrm{kg} / \mathrm{height}$ in $\mathrm{m}^{2}$ ) $\geq 95$ th percentile of the WHO 2007 growth-reference for 5-19 years $^{(6)}$. IR was assessed through homeostatic model assessment for IR which is a valid tool for evaluating IR in children and adolescents ${ }^{(7)}$. Homeostatic model assessment for IR $>3 \cdot 16$ was used as a threshold for $\mathrm{IR}^{(8)}$. Furthermore, pubertal maturation was determined using Tanner-Marshall descriptive standards by the same paediatric endocrinologist.

Exclusion criteria were following a diet and/or taking medications as the obesity and/or IR treatment, fasting glucose greater than $5.55 \mathrm{mmol} / \mathrm{l}$, having any metabolic, endocrine or gastrointestinal disease, physical disability limiting their physical activity, using tobacco, alcohol or any medications, practicing endurance sports and hypersensitivity for the food products under study. Two randomly assigned participants withdrew from the trial, and five were not included to the analysis of data because of several reasons (Fig. 1), which left fifteen participants assessed for the main outcomes.

\section{Study design and procedure}

This was a randomised, single-blind, cross-over clinical trial conducted on obese adolescents served two different test meals with the following similar GI and different II amounts: a low GI and low II (LGI-LII) content, and a low GI and high II content (LGI-HII). The order of the test meals was determined by using a computer-generated randomisation sequence before recruitment. The primary outcome was the postprandial response of serum glucose and insulin. Serum C-peptide levels, appetite sensations and subsequent food intakes were the secondary outcomes.

Participants received each test meal on two different mornings separated by a washout period of 1 week when they were asked

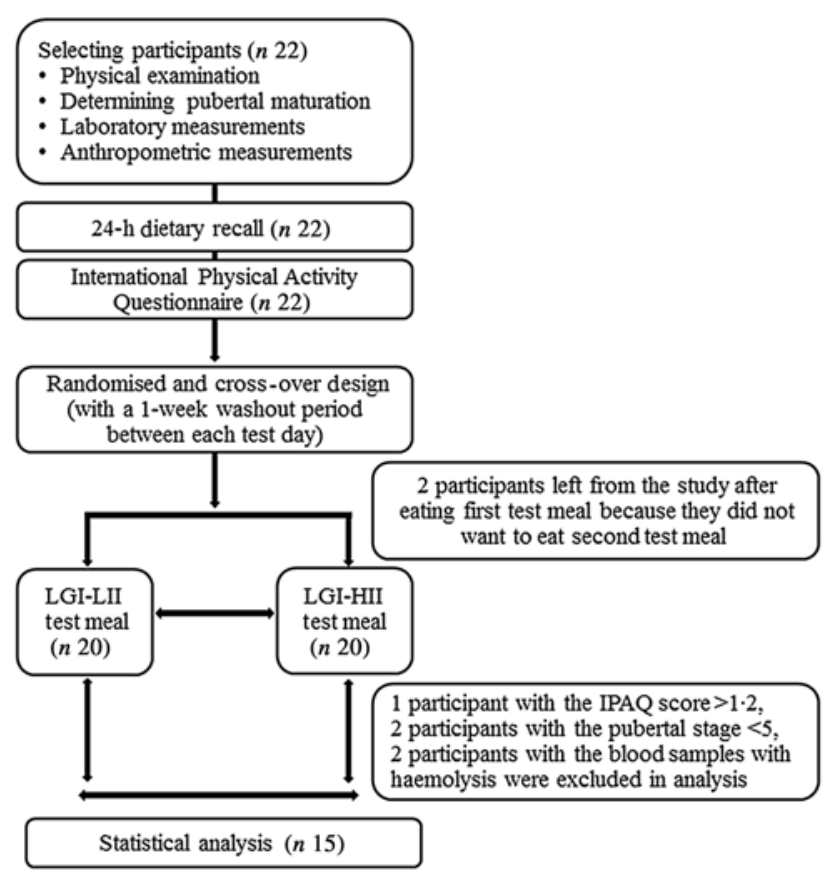

Fig. 1. Participant recruitment flow diagram. LGI-LII, low glycaemic index and low insulin index; LGI-HII, low glycaemic index and high insulin index; IPAQ, International Physical Activity Questionnaire.

to maintain their usual diet and physical $\operatorname{activity}^{(9)}$. On the day before each test meal, participants were instructed to eat a standard evening meal at 20.00 hours and to refrain from eating and/or drinking (except for water) and/or doing any physical activity beyond that of their typical daily activities ${ }^{(10)}$. Moreover, female participants were tested within the follicular phase of their menstrual cycle (3-10 d after onset of menses) to avoid possible influences of menstrual cycle phase on hormonal changes ${ }^{(11)}$.

On each testing day, participants arrived in the testing room at 08.00 hours following a 12 -h fast, and anthropometric measurements were completed before eating the test meal. Also, a catheter was introduced in an antecubital vein by a registered nurse, and a first blood sample was immediately drawn for baseline measurements ${ }^{(12)}$. At 08.30 hours participants received the test meal blinded to its nutritional characteristics and were asked to consume within $15 \mathrm{~min}^{(13,14)}$. The first bite in the mouth was set as time 0 , and the second blood sample was taken conventionally at exactly $15 \mathrm{~min}$ afterwards. During the postprandial period, participants remained at rest in the testing room, and other blood samples were obtained at 30, 45, 60, 90, 120, 180, and $240 \mathrm{~min}$ from the beginning of the meal ${ }^{(13)}$. Appetite scale was also applied at same time points ${ }^{(15)}$. Moreover, participants were asked to assess the palatability (visual appeal, smell, taste, aftertaste and overall palatability) of the test meal by visual analogue scale at $15 \mathrm{~min}$ (immediately after consuming the test meal) ${ }^{(16)}$ (online Supplementary Fig. S1). No food or drink other than water was allowed following consumption of the test meal until the ad libitum lunch. Water was available ad libitum throughout the first trial; however, the volume consumed was measured, and the participants drank the same volume during the second trial ${ }^{(17)}$. Participants were permitted to watch movies, read, or play with electronic devices (laptop computer, mobile 
Table 1. Nutritional composition, glycaemic index (GI) and insulin index (II) of the component foods in test meals

\begin{tabular}{|c|c|c|c|c|c|c|c|c|}
\hline & Weight (g) & Energy (kJ) & $\mathrm{AvCHO}^{*}(\mathrm{~g})$ & Protein $(\mathrm{g})$ & Fat $(\mathrm{g})$ & Fibre $(\mathrm{g})$ & GI (\%) & II (\%) \\
\hline \multicolumn{9}{|l|}{ LGI-LII } \\
\hline Grain bread & 25 & 217 & $9 \cdot 6$ & $2 \cdot 4$ & 0.4 & 1.7 & 50 & 41 \\
\hline Egg (boiled) & 42 & 246 & 0.0 & $5 \cdot 4$ & $4 \cdot 2$ & 0.0 & 0 & 23 \\
\hline Milk (full-fat) & 210 & 550 & $10 \cdot 4$ & $6 \cdot 0$ & $7 \cdot 1$ & 0.0 & 31 & 24 \\
\hline Breakfast cereal & 40 & 597 & 23.9 & 5.4 & $2 \cdot 4$ & $4 \cdot 2$ & 30 & 23 \\
\hline Apple & 200 & 469 & $24 \cdot 9$ & 0.9 & 0.9 & 3.8 & 36 & 43 \\
\hline Total & & 2079 & $68 \cdot 8$ & $20 \cdot 2$ & $15 \cdot 1$ & 9.7 & 35 & 30 \\
\hline \multicolumn{9}{|l|}{ LGI-HII } \\
\hline Grain bread & 18 & 157 & 6.9 & $1 \cdot 7$ & 0.3 & $1 \cdot 3$ & 50 & 41 \\
\hline Cheddar cheese & 22 & 373 & 0.0 & $5 \cdot 6$ & 7.5 & 0.0 & 0 & 33 \\
\hline Yogurt (low-fat strawberry) & 350 & 1383 & $54 \cdot 0$ & $13 \cdot 8$ & $6 \cdot 9$ & $4 \cdot 2$ & 31 & 84 \\
\hline Banana & 45 & 146 & 8.0 & 0.3 & 0.1 & 0.7 & 52 & 59 \\
\hline Total & & 2059 & $68 \cdot 8$ & 21.4 & $14 \cdot 8$ & $6 \cdot 2$ & 35 & 70 \\
\hline
\end{tabular}

AvCHO, available carbohydrate; LGI-LII, low GI and low II; LGI-HII, low GI and high II.

* Available carbohydrate including sugars and starch, excluding fibre.

phone etc.) or undertake other similar sedentary activities throughout each test day; however, they were not allowed to sleep $^{(10)}$.

The present study was conducted according to the guidelines laid down in the Declaration of Helsinki. The Clinical Research Ethics Committee of the Erciyes University approved the protocol (2015/451) on 2 October 2015, and all participants gave written informed consent. In addition, it was registered at ClinicalTrials.gov as NCT03387293.

\section{Composition of test meals}

Test meals were matched for macronutrients, and GI however had a 2-fold difference in II (Table 1). All foods served at breakfast were prepared on the day of each test meal. GI and II of foods in test meals were estimated by using the GI and II for $1000-\mathrm{kJ}$ portions of food tables published by Bao et al. ${ }^{(18)}$, with glucose as the reference food. The average meal GI and II were calculated as follows ${ }^{(4)}$ :

$$
\begin{gathered}
\text { Meal GI }=\frac{\sum_{a=1}^{n}\left(\text { GI }_{a} \times \mathrm{AvCHO}_{a} \times \text { Frequency }_{a}\right)}{\sum_{a=1}^{n}\left(\mathrm{AvCHO}_{a} \times \text { Frequency }_{a}\right)} \\
\text { Meal II }=\frac{\sum_{a=1}^{n}\left(I_{a} \times \text { Energy }_{a} \times \text { Frequency }_{a}\right)}{\sum_{a=1}^{n}\left(\text { Energy }_{a} \times \text { Frequency }_{a}\right)}
\end{gathered}
$$

where $n$ is the number of foods consumed, $\mathrm{GI}_{a}$ is the GI for food $a, \mathrm{II}_{a}$ is the II for food $a, \mathrm{AvCHO}_{a}$ is the available carbohydrate content per serving of food $a$, Energy $_{a}$ is the energy content per serving of food $a$ and Frequency ${ }_{a}$ is the consumption frequency of one serving of food $a$ during the meal.

\section{Anthropometric measurements}

Body weight and height were measured by an automatic height gauge scale (DENSI GL150) sensitive to 10-200 kg $\pm 50 \mathrm{~g}$ and 90-200 cm $\pm 1 \mathrm{~mm}$. Waist, hip and neck circumferences were measured to the nearest $0 \cdot 1 \mathrm{~cm}$ using a non-elastic tape with the participants standing, with the face directed towards, shoulders relaxed, and the tape was positioned at a level parallel to the floor. Body fatness was estimated by the bio-electrical impedance analysis method, by a segmental body composition analyser, Tanita BC-418MA (Tanita Corporation).

\section{Dietary and physical activity assessments}

Participants' dietary intakes were assessed by a face-to-face 24-h multiple-pass recall method using a photographic atlas of food portion sizes to quantify the data in the beginning of the study and on the day of each test meal ${ }^{(19)}$. Diet composition was analysed by the BeBiS Nutrition Information System software version 7.2.

Physical activity level was evaluated by the International Physical Activity Questionnaire short form, a validated survey instrument ${ }^{(20)}$, in the beginning of the study. The seven-item International Physical Activity Questionnaire records selfreported physical activity in the last $7 \mathrm{~d}$. Responses were converted to Metabolic Equivalent Task minutes per week according to the International Physical Activity Questionnaire scoring protocol.

\section{Laboratory measurements}

Fasting blood glucose, insulin, total cholesterol, HDL, LDL, TAG, alanine aminotransferase and aspartate aminotransferase were measured after a 12-h fast. Biochemical parameters were determined by using enzymatic kits from Roche Diagnostics with a Roche Cobas $₫ 8000$ Modular Analyzer Series. Blood samples after collection were immediately centrifuged and assayed. Serum glucose was measured using a spectrophotometric method (Roche Cobas 8000 Modular Analyzer Series, c702 module), and serum insulin and C-peptide by an electrochemiluminescence immunoassay method (Roche Cobas® 8000 Modular Analyzer Series, e602 module) using Roche kits (reference no. for glucose: 05168791, insulin: 12017547, C-peptide: 03184897; Roche Diagnostics).

\section{Assessment of appetite sensations}

Subjective assessment of appetite sensations was performed by using a visual analogue scale composed of lines (of $100 \mathrm{~mm}$ in length) with words anchored at each end, expressing the most positive and the most negative rating ${ }^{(16)}$. Visual analogue scale was used to assess appetite scores (hunger, fullness, desire to eat and prospective food consumption), desire for 
specific food types (sweet, salty, savoury and fatty) and the palatability of test meals (visual appeal, smell, taste, after taste and overall palatability). Furthermore, the separate visual analogue scale components such as hunger, fullness, desire to eat and prospective food consumption were combined to produce an additional measure termed 'composite appetite score'. This validated average appetite score was calculated for each time point using the following equation: ((hunger + desire to eat + prospective food consumption $+(100-$ fullness)) $/ 4^{(21)}$

\section{Ad libitum lunch}

At 240 min after the test breakfast, participants were presented with an ad libitum lunch following appetite sensation measurement ${ }^{(10)}$. Participants selected food from a buffet-style meal consisting of a variety of foods from each food group (meatballs, chicken nuggets, pasta with tomato sauce, potato salad, carrot salad, yogurt, white bread, wholegrain bread, cookies, apple, mandarin and bananas) ${ }^{(9,10)}$ with bottled water and some fruit juices (black cherry juice and peach juice) as beverage choices ${ }^{(22)}$. A list of all the foods served at the buffet-style lunch including their energy and macronutrient composition per $100 \mathrm{~g}$ is detailed in online Supplementary Table S1. These foods were determined according to adolescents' preference to consume and frequent consumption in the Central Anatolia region and were widely preferred in similar studies. During the lunch, participants were left alone in a quiet room with controlled lightning and ambient room temperature, and asked to consume whatever they wanted and to eat until they felt comfortably full ${ }^{(10)}$. Foods were weighed or measured to the nearest $0 \cdot 1 \mathrm{~g}$ before consumption, and any remaining food was re-weighed to determine intake at lunch. Energy and macronutrient intakes were calculated using the National Food Composition Database (TurKomp) $)^{(23)}$ and manufacturer labelling. Moreover, the first food to start eating, all foods selected by participants and duration of meal were recorded at lunchtime.

\section{Statistics}

Sample size and power analysis. The study power was based on a difference in incremental AUC (iAUC) across the 4-h period for insulin $^{(24)}$. Power analysis using G*Power (version 3.1.9.4) revealed that the sample size of fifteen participants provided 82.8 power (effect size $=0.807, \alpha=0.05$ ) to detect differences between two iAUC of the insulin after consuming LGI-LII $(5182.9 \pm 3513.2 \mathrm{pmol} / \mathrm{l} \times \mathrm{min})$ and LGI-HII $(8053.6 \pm$ $3601.3 \mathrm{pmol} / \mathrm{l} \times \mathrm{min})$ test meals in obese adolescents with IR.

Data analysis. Statistical analysis was performed using the IBM SPSS Statistics (version 22.0) software. Data were expressed as the number $(n)$ and percentage (\%) for categorical variables, and mean and standard deviation for continuous variables. Normality was assessed using the histogram and normal Q-Q plots, and also Shapiro-Wilk test. Furthermore, continuous variables were examined for skewness and kurtosis, and logtransformed before analysis and reported back-transformed geometric mean $(G)$ and standard error when required ${ }^{(25)}$. The iAUC (negative area beneath the fasting level was ignored) for serum glucose, insulin and C-peptide as well as the total AUC for the subjective appetite ratings were calculated according to the trapezoidal rule ${ }^{(26)}$. A Student's two-tailed $t$ test for paired data was applied to determine statistical differences between $\mathrm{AUC}^{(14,24)}$. In addition, betweengroup comparisons were analysed by using two-factor (time $\times$ meal) repeated-measures ANOVA, and Bonferroni post hoc tests were applied to significant time $\times$ meal interactions ${ }^{(14,24)}$. Baseline values for each variable were compared between groups by using paired $t$ tests. Categorical variables were compared by the $\chi^{2}$ tests. Wilcoxon signed rank tests were used for continuous variables without normal distributions. For all statistical analyses, $P$ values less than 0.05 were considered to have statistical significance ${ }^{(27)}$.

\section{Results}

\section{Participants}

Baseline characteristics of fifteen participants (seven males, eight females) who completed the experimental protocol were presented in Table 2. Participants' dietary intakes assessed by a-24-h dietary recall were similar on the day of each test meal (online Supplementary Table S2). During the test days, all test meals were fully consumed, and no complaints or digestive disturbances were recorded.

\section{Postprandial glucose, insulin and C-peptide responses}

Fasting serum glucose, insulin and C-peptide levels were similar before the LGI-LII and LGI-HII meals $(P>0 \cdot 05)$. As predicted by the GI of test meals, there were no significant differences in the early postprandial glucose responses to the test meals $(P>0.05)$. However, the LGI-LII meal led to a borderline-significant $(P=0.062)$ and significant $(P=0.042)$ higher glucose responses than did the LGI-HII meal in the late and total postprandial period, respectively. On the other hand, the early, late and total postprandial C-peptide responses did not differ between the test meals $(P>0.05)$ (Fig. 2). Although there was no cross-over effect of test meal on the serum glucose (effect of time, $P<0 \cdot 001$; effect of meal, $P=0 \cdot 130$; interaction between time $\times$ meal, $P=0.449$ ) and C-peptide (effect of time, $P<0 \cdot 001$; effect of meal, $P=0.704$; interaction between time $\times$ meal, $P=0.703$ ) concentrations over $4 \mathrm{~h}$ period, the glucose level at $45 \mathrm{~min}$ was higher following the LGI-LII meal compared with the LGI-HII meal $(P=0 \cdot 028$, by post boc $t$ test).

Insulin responses to the meal challenge were more rapid and higher after the LGI-HII meal compared with the LGI-LII meal. During the early response ( $\mathrm{iAUC}_{0-30} \mathrm{~min}$ ), serum insulin was lower by $56.1 \%$ after the LGI-LII meal $v$. LGI-HII meal $(P=0 \cdot 007)$. After the LGI-LII meal, insulin iAUC $_{45-240} \mathrm{~min}$ and iAUC $_{0-240}$ min were 34.6 and $35.6 \%$ lower compared with the insulin responses after the LGI-HII meal $(P=0.004$ and $P=0.009$, respectively) (Fig. 2). Furthermore, a cross-over effect of test meal on the serum insulin concentration over $4 \mathrm{~h}$ period was detected (effect of time, $P<0.001$; effect of meal, $P=0.001$; interaction between time $\times$ meal, $P=0.001$ ), and at 30 and $120 \mathrm{~min}$, the insulin levels were lower following 
Table 2. Baseline characteristics of participants

(Number and percentage; mean values and standard deviations; geometric means with their standard errors; $n$ 15)

\begin{tabular}{|c|c|c|}
\hline Characteristics & Mean & SD \\
\hline \multicolumn{3}{|l|}{ Clinical characteristics } \\
\hline \multicolumn{3}{|l|}{ Male } \\
\hline$n$ & \multicolumn{2}{|c|}{7} \\
\hline$\%$ & \multicolumn{2}{|c|}{$46 \cdot 7$} \\
\hline Age (years) & $15 \cdot 0$ & 1.7 \\
\hline Systolic blood pressure $(\mathrm{kPa})$ & 14.74 & 1.82 \\
\hline Diastolic blood pressure $(\mathrm{kPa})$ & 8.89 & 1.48 \\
\hline \multicolumn{3}{|l|}{ Anthropometric characteristics } \\
\hline BMI Z-score (sD) & $3 \cdot 12$ & 0.99 \\
\hline \multicolumn{3}{|l|}{ Male } \\
\hline Waist circumference $(\mathrm{cm})$ & $102 \cdot 71$ & 5.80 \\
\hline Hip circumference $(\mathrm{cm})$ & 114.29 & 3.60 \\
\hline Neck circumference $(\mathrm{cm})$ & 38.99 & 1.94 \\
\hline Body fat $(\%)$ & $32 \cdot 51$ & 3.82 \\
\hline Trunk fat (\%) & 28.87 & 2.88 \\
\hline \multicolumn{3}{|l|}{ Female } \\
\hline Waist circumference (cm) & $104 \cdot 23$ & $12 \cdot 41$ \\
\hline Hip circumference $(\mathrm{cm})$ & $120 \cdot 49$ & $15 \cdot 68$ \\
\hline Neck circumference $(\mathrm{cm})$ & 37.65 & 2.33 \\
\hline Body fat $(\%)$ & $42 \cdot 23$ & $6 \cdot 65$ \\
\hline Trunk fat (\%) & 36.64 & 7.58 \\
\hline \multicolumn{3}{|l|}{ Laboratory characteristics } \\
\hline Fasting blood glucose $(\mathrm{mmol} / \mathrm{l})$ & 4.82 & 0.64 \\
\hline Fasting blood insulin (pmol/l) & $157 \cdot 29$ & 60.93 \\
\hline HOMA-IR & 4.62 & 1.69 \\
\hline Serum total cholesterol $(\mathrm{mmol} / \mathrm{l})$ & 4.02 & 1.21 \\
\hline Serum HDL-cholesterol (mmol/l) & $1 \cdot 11$ & 0.19 \\
\hline Serum LDL-cholesterol (mmol/l) & $2 \cdot 21$ & 0.91 \\
\hline Serum TAG $(\mathrm{mmol} / \mathrm{l})$ & 1.48 & 0.75 \\
\hline Serum alanine aminotransferase ( $\mu$ kat $/ \mathrm{l})$ & 0.36 & 0.14 \\
\hline Serum aspartate aminotransferase ( $\mu \mathrm{kat} / \mathrm{l})$ & 0.31 & 0.08 \\
\hline \multicolumn{3}{|l|}{ Dietary intake* } \\
\hline \multicolumn{3}{|l|}{ Male } \\
\hline \multicolumn{3}{|l|}{ Energy $(\mathrm{kJ} / \mathrm{d})$} \\
\hline Geometric mean & \multirow{2}{*}{\multicolumn{2}{|c|}{$\begin{array}{c}6693 \cdot 1 \\
532 \cdot 2\end{array}$}} \\
\hline SE & & \\
\hline Carbohydrate (E\%) & 53.71 & 10.48 \\
\hline Protein $(g)$ & 53.93 & $10 \cdot 29$ \\
\hline Fat $(E \%)$ & $32 \cdot 36$ & $9 \cdot 32$ \\
\hline Fibre $(\mathrm{g})$ & $15 \cdot 82$ & 3.41 \\
\hline \multicolumn{3}{|l|}{ Female } \\
\hline \multicolumn{3}{|l|}{ Energy $(\mathrm{kJ} / \mathrm{d})$} \\
\hline Geometric mean & \multirow{2}{*}{\multicolumn{2}{|c|}{$\begin{array}{c}6656.5 \\
737.8\end{array}$}} \\
\hline SE & & \\
\hline Carbohydrate (E\%) & 47.88 & 6.09 \\
\hline Protein $(g)$ & 47.89 & $15 \cdot 96$ \\
\hline Fat $(\mathrm{E} \%)$ & $40 \cdot 38$ & 4.81 \\
\hline Fibre $(\mathrm{g})$ & $18 \cdot 17$ & 5.81 \\
\hline \multicolumn{3}{|l|}{ Total MET (min/week) } \\
\hline \multicolumn{3}{|l|}{ Male } \\
\hline Geometric mean & \multicolumn{2}{|c|}{$606 \cdot 20$} \\
\hline & \multicolumn{2}{|c|}{183.51} \\
\hline \multicolumn{3}{|l|}{ Female } \\
\hline Geometric mean & & \\
\hline SE & & \\
\hline
\end{tabular}

HOMA-IR, homeostatic model assessment for insulin resistance; MET, metabolic equivalent task.

* Determined from the 24-h dietary recalls using BeBiS Nutrition Database software version 7.2

the LGI-LII meal than following the LGI-HII meal $(P=0 \cdot 002$ and $P=0.032$, by post boc $t$ test). Additionally, peak insulin secretion occurred at $30 \mathrm{~min}$ after the LGI-HII meal while it was reaching the peak level at $45 \mathrm{~min}$ after the LGI-LII meal. However, patterns of C-peptide levels were a great contrast to those of insulin with a more rapid peak after the LGI-LII meal than the LGI-HII meal ( $45 \mathrm{~min} v .60 \mathrm{~min}$ ), and serum glucose peaked at 30 min following both of the two test meals.

\section{Appetite sensations}

The postprandial appetite sensations after each test meal were shown in Table 3. Baseline measures for all parameters were similar on both test days $(P>0.05)$. No differences were found in the postprandial appetite sensations between test days, except the feeling of hunger. After the LGI-LII meal, the late and total AUC for hunger was 25.8 and $27.5 \%$ lower compared with the after the LGI-HII meal $(P<0.05)$ (Fig. 2). There was also no cross-over effect of test meals on appetite scores over $4 \mathrm{~h}$ period (effect of time, $P<0.05$ for all ratings; effect of meal, $P>0.05$ for all ratings; interaction between time $\times$ meal, $P>0.05$ for all ratings), except the effect of meal on hunger score (effect of time, $P<0.001$; effect of meal, $P=0.010$; interaction between time $\times$ meal, $P>0 \cdot 05)$.

\section{Food intake at ad libitum lunch}

Energy and macronutrient intakes at the ad libitum buffet-style lunch on each test days are shown in online Supplementary Table S3. There was no evidence that consuming the LGI-LII breakfast decreased energy intake from the buffet lunch meal $(4853.7 \pm 319 \cdot 2 \mathrm{~kJ} v .4882 \cdot 9 \pm 300 \cdot 3 \mathrm{~kJ}, P=0.897)$. There was also no significant difference in the macronutrient composition and dietary fibre of the foods chosen from the buffet between the two test meals $(P>0 \cdot 05)$. Moreover, the first food consumed at each ad libitum lunch was examined, and there was no evidence of a change in food preference when the II of breakfast was altered $\left(P=0.449\right.$ by $\chi^{2}$ test $)$.

\section{Palatability of the test meals}

Ratings for visual appeal, taste, smell, after taste and overall palatability after the two test meals are presented in Fig. 3. Apparently, the ratings for after taste and visual appeal were similar after the LGI-LII and LGI-HII test meals on the two test days $(P>0 \cdot 05)$. However, the LGI-LII meal had lower scores for taste, smell and overall palatability compared with the LGI-HII meal $(P<0 \cdot 05)$.

\section{Discussion}

The present study provides the first evidence of the effect of II in the context of a mixed meal on metabolic parameters in obese adolescents with IR. The findings of the present randomised, cross-over clinical trial have demonstrated that a 2 -fold difference in the calculated insulin demand (based on the II of component foods) led to a significant difference in the postprandial response for insulin over $4 \mathrm{~h}$, even when macronutrients and GI were matched between two meals. This result suggests that a low-II diet may reduce nutrient-induced hyperinsulinaemia and lead to a $\mathrm{s}$ ignificantly greater improvement in IR and sensitivity than the high-II diet in obese adolescents with IR. 
Table 3. Postprandial appetite sensations after each test meal

(Geometric means $(\mathrm{G})$ with their standard errors, $n$ 15)

\begin{tabular}{|c|c|c|c|c|c|c|}
\hline \multirow[b]{2}{*}{$\operatorname{AUC}(\mathrm{mm} \times \mathrm{min})$} & \multicolumn{2}{|c|}{ LGI-LII } & \multicolumn{2}{|c|}{ LGI-HII } & \multirow[b]{2}{*}{$P$} & \multirow[b]{2}{*}{ Difference (\%) } \\
\hline & $\mathrm{G}$ & SE & G & SE & & \\
\hline Hunger & $6409 \cdot 8$ & $870 \cdot 6$ & $8174 \cdot 1$ & $976 \cdot 3$ & $0.037^{*}$ & $-27 \cdot 5$ \\
\hline Fullness & $12767 \cdot 0$ & $1177 \cdot 5$ & $12192 \cdot 1$ & $1118 \cdot 0$ & 0.658 & 4.5 \\
\hline Desire to eat & $6762 \cdot 8$ & 940.9 & $7011 \cdot 1$ & $1065 \cdot 1$ & 0.669 & $-3 \cdot 7$ \\
\hline Prospective food consumption & $6548 \cdot 3$ & $808 \cdot 3$ & $6704 \cdot 4$ & 984.4 & 0.791 & -2.4 \\
\hline Composite appetite score & $7355 \cdot 1$ & $863 \cdot 0$ & $8189 \cdot 6$ & $939 \cdot 7$ & 0.257 & $-11 \cdot 3$ \\
\hline Desire for sweet food & $3758 \cdot 1$ & $1029 \cdot 9$ & $4394 \cdot 7$ & $1313 \cdot 2$ & 0.228 & $-16 \cdot 9$ \\
\hline Desire for salty food & $1628 \cdot 9$ & 955.9 & $1171 \cdot 1$ & $822 \cdot 2$ & 0.189 & $28 \cdot 1$ \\
\hline Desire for savoury food & $1000 \cdot 9$ & $586 \cdot 4$ & 1140.9 & $853 \cdot 1$ & 0.617 & $-14 \cdot 0$ \\
\hline Desire for fatty food & $1245 \cdot 3$ & $996 \cdot 2$ & 1361.9 & 933.3 & 0.589 & $-9 \cdot 4$ \\
\hline
\end{tabular}

LGI-LII, low glycaemic index and low insulin index; LGI-HII, low glycaemic index and high insulin index.

* Student's two-tailed $t$ test for paired data $(P<0.05)$.

(a)

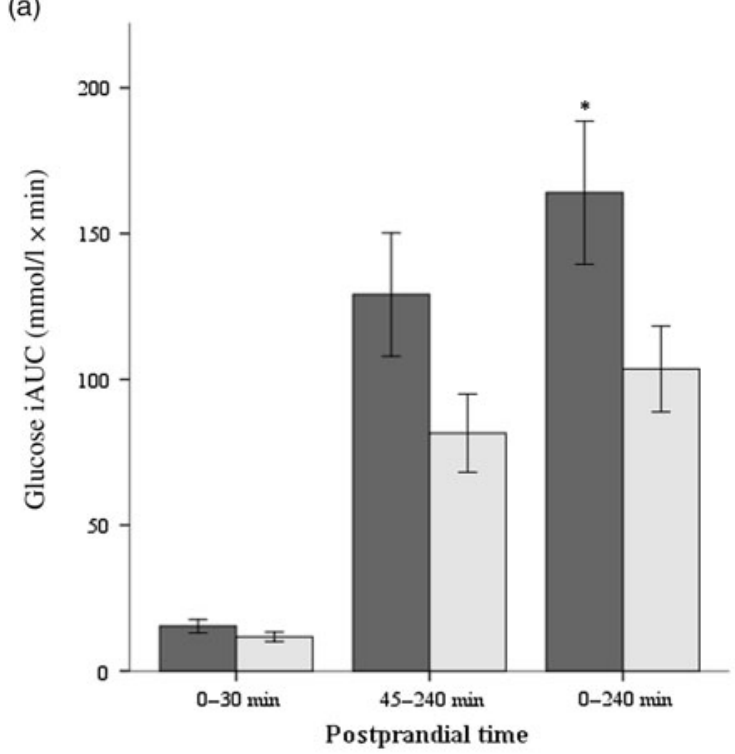

(b)

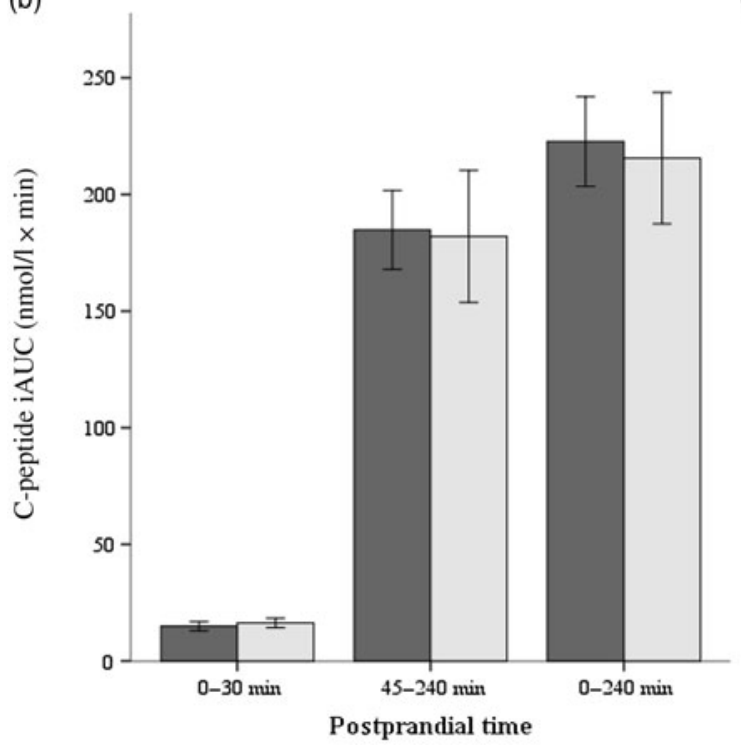

(c)

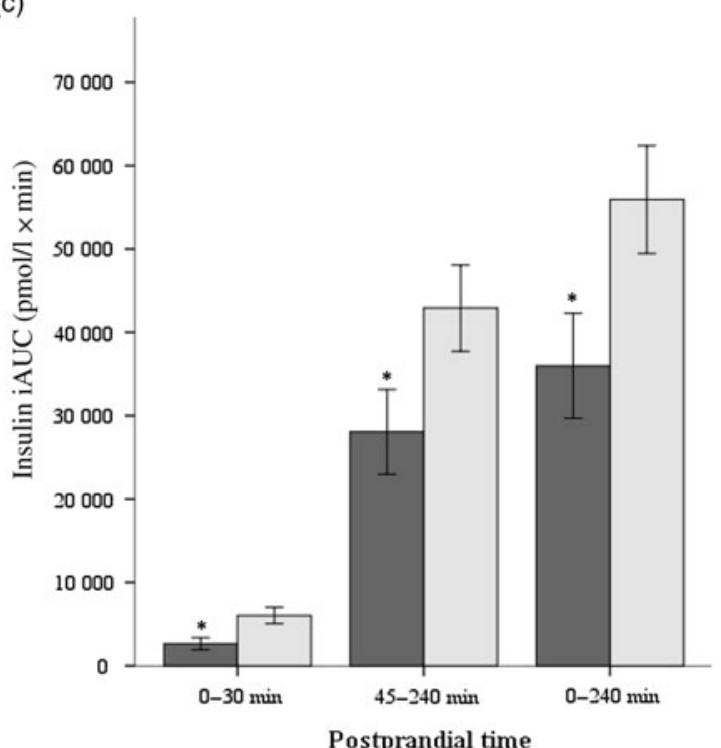

(d)

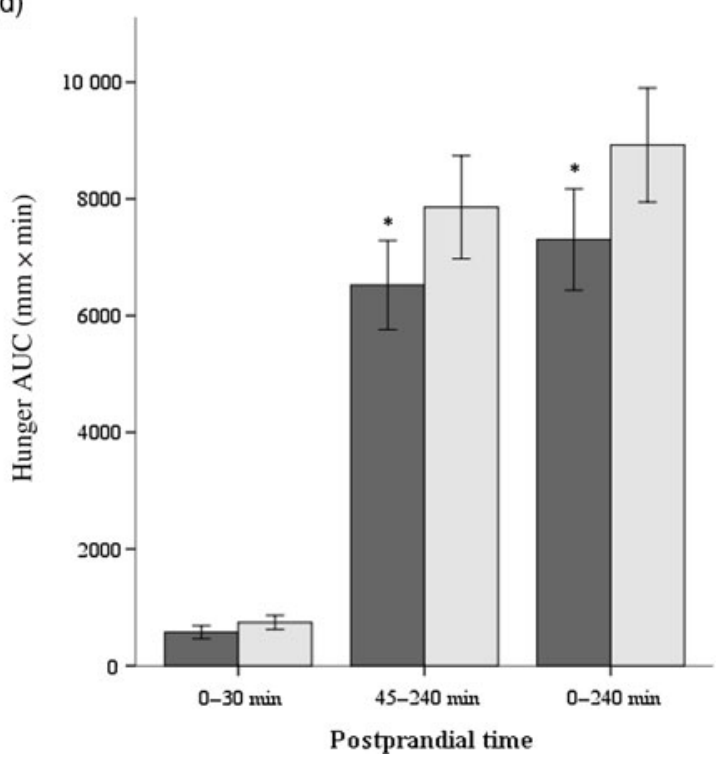

Fig. 2. Early, late and total postprandial metabolic and appetite responses after each test meal over $4 \mathrm{~h}(n$ 15): (a) glucose, (b) insulin, (c) C-peptide and (d) hunger. $\square$, Low glycaemic index and low insulin index; $\square$, low glycaemic index and high insulin index; iAUC, incremental AUC. Values are means, with their standard errors represented by vertical bars. ${ }^{*} P<0.05$ (Student's two-tailed $t$ test for paired data). 


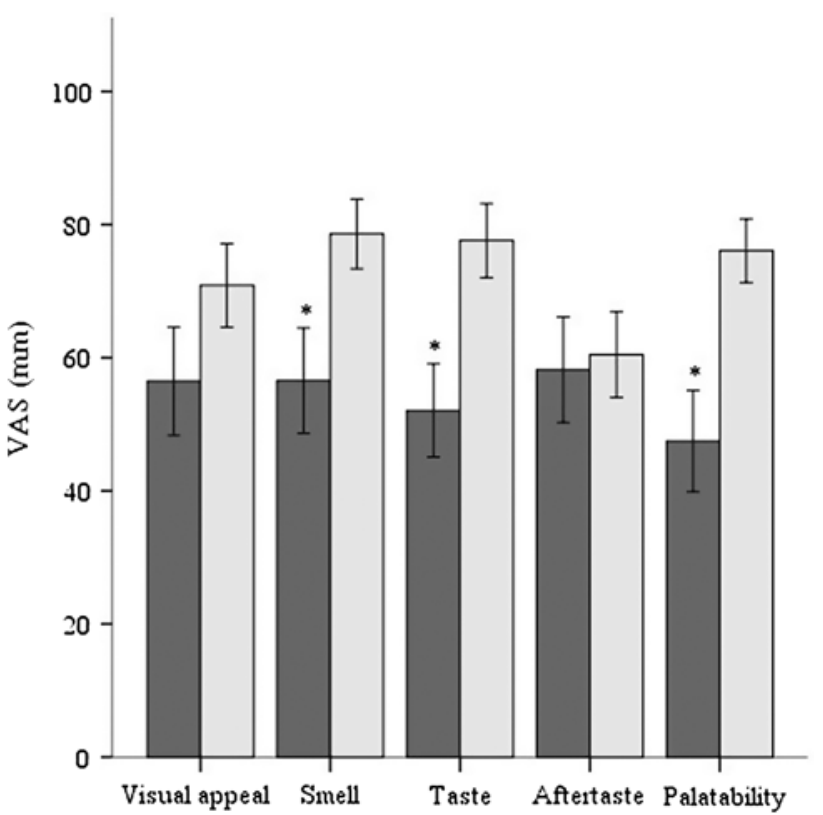

Fig. 3. Palatability scores of test meals ( $n$ 15). $\square$, Low glycaemic index and low insulin index; $\square$, low glycaemic index and high insulin index; VAS, visual analogue scale. Values are means, with their standard errors represented by vertical bars. ${ }^{*} P<0.05$ (Wilcoxon signed rank tests).

A low-GI diet recommended as an alternative dietary intervention in the treatment of obesity could also help prevent the development of impaired glucose homeostasis ${ }^{(28)}$. Physiological mechanisms that associate high GI and weight gain are based on the postprandial metabolic environment initiated by hyperglycaemia and hyperinsulinaemia, which accelerate glucose oxidation and stimulate fat storage ${ }^{(29)}$. However, insulin secretion may also be stimulated by dietary protein and fat, while GI is only associated with the effect of carbohydrate-containing foods on postprandial responses ${ }^{(5)}$. For this reason, it may be beneficial to use the II value, which directly quantifies the postprandial insulin response to any food relative to a reference food (glucose) and allows for assessing the effect of all nutrients, not just carbohydrates ${ }^{(4)}$. The first study in the literature providing an evidence of the physiological validity of II in the context of mixed meals was published in healthy and diabetic adults $^{(24)}$. To our knowledge, the present study is the second research of its kind contributing to the literature on the effect of meal II on postprandial responses, and is the first research conducted in obese paediatric subjects with IR. In the present study, consistent with the first study by Bell et $a l^{(24)}$, the postprandial insulin responses were higher after the LGI-HII meal compared with the LGI-LII meal. The obtained data have demonstrated that the meal II may affect the postprandial insulin response independently of GI.

In the early postprandial period, there was no significant difference in the postprandial glucose responses between the test meals, while the postprandial insulin response after the LGI-HII meal was higher than after the LGI-LII meal in the present study. Accordingly, differences in glycaemia cannot hold responsible for the observed differences in insulinaemic responses. The effect may be due to different types of fat and protein. Even though almost all protein types may appear to mediate an insulinotropic effect, certain proteins and amino acids, such as lysine, threonine, leucine, isoleucine and valine, have been demonstrated to modulate insulin response ${ }^{(26)}$. Fatty acids also have a potential effect on insulin secretion by correlating with the fatty acid chain length and degree of saturation; for instance, long-chain unsaturated fatty acids have been shown to increase insulin response ${ }^{(24)}$.

On the other hand, contrary to the study by Bell et al. ${ }^{(24)}$, there was a difference in the total postprandial glucose responses in the present study. When the time $\times$ meal effect on postprandial glucose and insulin concentrations were examined, serum insulin level at 30 min was higher after the LGI-HII $v$. LGI-LII meal, and then serum glucose level at $45 \mathrm{~min}$ was lower after the LGIHII $v$. LGI-LII meal. The decreased postprandial glucose response after LGI-HII can therefore be attributed to the early postprandial hyperinsulinaemia, which has an immediate effect on plasma glucose concentrations. However, to prevent blood glucose concentrations from falling as insulin concentration increases, hormones acting in opposition to insulin increase hepatic glucose output to maintain blood glucose concentrations within the normal range ${ }^{(24)}$. Hence, it may be predicted that the responses of glucagon and other glucoregulatory hormones, such as incretins, would differ in the late postprandial period, thereby being similar glucose levels ${ }^{(14,24)}$. Consequently, methodological differences (different postprandial period, $4 \mathrm{~h} v .8 \mathrm{~h}$; different washout period, 1 week $v$. none) may be why the results of the present study on postprandial glucose responses were inconsistent with previous findings by Bell et al. ${ }^{(24)}$. This assumption needs to be confirmed in additional studies to focus on mechanisms linking II with postprandial responses.

We came across only one cross-sectional study in the literature to examine the relationship between dietary II and serum C-peptide levels, and no correlation was demonstrated between them in the present study ${ }^{(4)}$. Furthermore, no clinical studies have been found that investigate the effect of the meal II on the postprandial C-peptide response. However, the results of the present study have supported that data from the crosssectional study, and no differences were found in the postprandial C-peptide responses between the LGI-LII and LGI-HII meals. Although there was a significant difference in early postprandial insulin response after the high-II meal, that increase did not persist in the later period, and there was no difference in C-peptide response. This result has given rise to thought about that a high-II meal may lead to the use of pancreatic insulin reservoirs that provide rapid insulin response; however, this may not affect insulin production. Consequently, there will be no effect on C-peptide production. As known, the major stimulant of insulin secretion is carbohydrates, and the GI is a criterion that indicates carbohydrate quality; however, the II concept allows for the testing of all nutrients, including proteins and fats ${ }^{(4)}$. In the present study, the possible effects of carbohydrates were minimised by the matched GI of the prepared test meals. Thus, it is not surprising that such a result has been achieved. Nevertheless, this conclusion does not make the II value of a meal less important than the GI. Hyperinsulinaemia in the early postprandial period after a high-II meal may reduce the insulin sensitivity of peripheral tissues and may exacerbate present IR and also contribute to the 
development of type 2 diabetes by inducing a decrease in pancreatic insulin reserve ${ }^{(4,24)}$.

To our knowledge, this is the first study to investigate the effect of meal II on appetite and subsequent food intake. Results of the present study showed that the LGI-LII meal reduced the perceived hunger more effectively than did the LGI-HII meal. However, the LGI-LII and LGI-HII meals were not observed to have different effects on subsequent food intake. Although the suggested mechanisms for the possible effects of II on appetite are present, clinical trials supporting these mechanisms are based on only single-food studies $^{(30-33)}$. The higher hunger rating after the high-II meal obtained in the present study may be related to the increased insulin and decreased glucose responses ${ }^{(2)}$. However, further clinical trials are required to support these results. On the other hand, it has been suggested that a decrease in total energy consumption during a day or a period of a few days may occur with decreased insulin response and a prolonged satiety, even if there was no difference in energy intake at a subsequent meal $^{(34)}$. Similarly, in the present study, possible beneficial effects of low II are also valid for our results, if a low-II meal is considered to be able to cause less insulin response and to have lower total hunger score at the end of $4 \mathrm{~h}$. Moreover, participants' consumption of lunch at the hospital may have affected ad libitum energy intake, and different outcomes may be obtained in their familiar environment.

The present study has the strength of evaluating metabolic responses to breakfasts that correspond to a commonplace meal instead of a single food. This concept provides additional practical clinical relevance on the results of the present study. In addition, the test meals differed only in their II amount and were constructed to obtain a 2-fold difference in insulin demand as predicted by the II of the component foods. This difference is quite similar to that used in the unique study comparing the effect of low- $v$. high-II in the context of mixed meals ${ }^{(24)}$. Furthermore, the present study has achieved the strict control for potential confounders like pubertal stage, metabolic conditions, physical activity level, menstrual cycle stage and dietary intake on the previous day. Nonetheless, there were some limitations of the present study. The first limitation is that the participants consumed both test meals at the hospital setting, and hence the results may differ from what can be realistically achieved in the free-living state. The second limitation is that despite subjective assessment of appetite sensations, we did not investigate the effect of meal II on appetite hormone such as peptide YY, neuropeptide Y, cholecystokinin, ghrelin. Therefore, it is unknown whether the meal II may affect the appetite hormones in the postprandial period. However, the result of present study may lead to initiate other studies to determine the association between II and metabolic parameters to play a role in the regulation of appetite.

In conclusion, the present study has shown that in obese adolescents with IR consumption of a high-II meal resulted a less favourable postprandial response as evidenced by increased insulin response than after consuming a low-II meal, even when the meals were matched for energy, macronutrients and GI. The present study has also demonstrated that only II independent of GI or macronutrients in the context of a mixed meal could affect the perceived hunger in obese adolescents with IR. Overall, the calculation of II of meals or diets may be a useful dietary approach to reduce postprandial hyperinsulinaemia and the perceived hunger in obese adolescents with IR. These findings may assist in developing new dietary interventions for obese adolescents with IR, but this assumption needs to be confirmed in further short- and/or long-term clinical trials.

\section{Acknowledgements}

We are extremely grateful to all the adolescents and families who took part in the present study, and the whole paediatric endocrinology team, which includes doctors, nurses, medical staff and clerical staff. We also thank Ferhan Elmali (associate professor at Izmir Katip Celebi University, Faculty of Medicine, Department of Biostatistics) for his help with statistical analyses.

The project was funded by a grant from the Scientific and Technological Research Council of Turkey (TUBITAK) within '1002 - Short Term R\&D Funding Program' with a project number of 116 S069. Z. C. was supported by a 2211/A - National Doctoral Fellowship 2013-2016 from TUBITAK and an ESPEN 2017 Travel Fellowship Award from 39. ESPEN Congress Scientific Committee.

Z. C. designed the research protocol; conducted the research and statistical analysis; prepared the manuscript and had primary responsibility for final content. N. H. contributed to the planning and management of study, laboratory and data analyses and conducted the research. H. G. O. designed the study and contributed to the manuscript writing. All authors read and approved the final manuscript.

The authors declare no conflict of interest.

\section{Supplementary material}

For supplementary materials referred to in this article, please visit https://doi.org/10.1017/S0007114519001351

\section{References}

1. Murakami K, McCaffrey TA, Gallagher AM, et al. (2014) Dietary glycemic index and glycemic load in relation to changes in body composition measures during adolescence: Northern Ireland Young Hearts Study. Int J Obes (Lond) 38, 252-258.

2. Joslowski G, Halim J, Goletzke J, et al. (2015) Dietary glycemic load, insulin load, and weight loss in obese, insulin resistant adolescents: RESIST study. Clin Nutr 34, 89-94.

3. Bao J, de Jong V, Atkinson F, et al. (2009) Food insulin index: physiologic basis for predicting insulin demand evoked by composite meals. Am J Clin Nutr 90, 986-992.

4. Nimptsch K, Brand-Miller JC, Franz M, et al. (2011) Dietary insulin index and insulin load in relation to biomarkers of glycemic control, plasma lipids, and inflammation markers. Am J Clin Nutr 94, 182-190.

5. Joslowski G, Goletzke J, Cheng G, et al. (2012) Prospective associations of dietary insulin demand, glycemic index, and glycemic load during puberty with body composition in young adulthood. Int J Obes (Lond) 36, 1463-1471.

6. World Health Organization (2007) The challenge of obesity in the WHO European Region and the strategies for response. 
http://www.who.int/growthref/who2007_bmi_for_age/en/ (accessed October 2016).

7. Keskin M, Kurtoglu S, Kendirci M, et al. (2005) Homeostasis model assessment is more reliable than the fasting glucose/ insulin ratio and quantitative insulin sensitivity check index for assessing insulin resistance among obese children and adolescents. Pediatrics 115, e500-e503.

8. Karatzi K, Moschonis G, Barouti AA, et al. (2014) Dietary patterns and breakfast consumption in relation to insulin resistance in children. The Healthy Growth Study. Public Health Nutr 17, 2790-2797.

9. Mehrabani S, Safavi SM, Mehrabani S, et al. (2016) Effects of low-fat milk consumption at breakfast on satiety and short-term energy intake in 10- to 12-year-old obese boys. Eur J Nutr $\mathbf{5 5}$, 1389-1396.

10. Baum JI, Gray M \& Binns A (2015) Breakfasts higher in protein increase postprandial energy expenditure, increase fat oxidation, and reduce hunger in overweight children from 8 to 12 years of age. J Nutr 145, 2229-2235.

11. Chowdhury EA, Richardson JD, Tsintzas K, et al. (2016) Effect of extended morning fasting upon ad libitum lunch intake and associated metabolic and hormonal responses in obese adults. Int J Obes (Lond) 40, 305-311.

12. Bidwell AJ, Fairchild TJ, Wang L, et al. (2014) Effect of increased physical activity on fructose-induced glycemic response in healthy individuals. Eur J Clin Nutr 68, 1048-1054.

13. Runchey SS, Valsta LM, Schwarz Y, et al. (2013) Effect of lowand high-glycemic load on circulating incretins in a randomized clinical trial. Metabolism 62, 188-195.

14. Jakubowicz D, Wainstein J, Ahrén B, et al. (2015) High-energy breakfast with low-energy dinner decreases overall daily hyperglycaemia in type 2 diabetic patients: a randomised clinical trial. Diabetologia 58, 912-919.

15. Yip W, Wiessing KR, Budgett $S$, et al. (2013) Using a smaller dining plate does not suppress food intake from a buffet lunch meal in overweight, unrestrained women. Appetite 69, 102-107.

16. Flint A, Raben A, Blundell JE, et al. (2000) Reproducibility, power and validity of visual analogue scales in assessment of appetite sensations in single test meal studies. Int $J$ Obes Relat Metab Disord 24, 38-48.

17. Zakrzewski JK, Stevenson EJ \& Tolfrey K (2012) Effect of breakfast glycemic index on metabolic responses during rest and exercise in overweight and non-overweight adolescent girls. Eur J Clin Nutr 66, 436-442.

18. Bao J, Atkinson F, Petocz P, et al. (2011) Prediction of postprandial glycemia and insulinemia in lean, young, healthy adults: glycemic load compared with carbohydrate content alone. Am J Clin Nutr 93, 984-996.

19. Warren JM, Henry CJ \& Simonite V (2003) Low glycemic index breakfasts and reduced food intake in preadolescent children. Pediatrics 112, e414.

20. Saglam M, Arikan H, Savci S, et al. (2010) International physical activity questionnaire: reliability and validity of the Turkish version. Percept Mot Skills 111, 278-284.
21. Zafar TA, Kabir Y \& Ghazaii C (2011) Low glycemic index foods suppress glycemic response, appetite and food intake in young Kuwaiti females. Kuwait J Sci Eng 38, 111-123.

22. Aston LM, Stokes CS \& Jebb SA (2008) No effect of a diet with a reduced glycaemic index on satiety, energy intake and body weight in overweight and obese women. Int J Obes (Lond) 32, $160-165$.

23. The Scientific and Technological Research Council of Turkey (2014) National food composition database TürKomp. turkomp.gov.tr (accessed November 2015).

24. Bell KJ, Bao J, Petocz P, et al. (2015) Validation of the food insulin index in lean, young, healthy individuals, and type 2 diabetes in the context of mixed meals: an acute randomized crossover trial. Am J Clin Nutr 102, 801-806.

25. Akin L, Kendirci M, Narin F, et al. (2015) The endocrine disruptor bisphenol A may play a role in the aetiopathogenesis of polycystic ovary syndrome in adolescent girls. Acta Paediatr 104, e171-e177.

26. Gunnerud UJ, Heinzle C, Holst JJ, et al. (2012) Effects of pre-meal drinks with protein and amino acids on glycemic and metabolic responses at a subsequent composite meal. PLOS ONE 7, e44731.

27. Caferoglu Z, Inanc N, Hatipoglu N, et al. (2016) Health-related quality of life and metabolic control in children and adolescents with type 1 diabetes mellitus. J Clin Res Pediatr Endocrinol 8, 67-73.

28. Mirza NM, Klein CJ, Palmer MG, et al. (2011) Effects of high and low glycemic load meals on energy intake, satiety and hunger in obese Hispanic-American youth. Int $J$ Pediatr Obes 6, e523-e 531.

29. Juanola-Falgarona M, Salas-Salvado J, Ibarrola-Jurado $\mathrm{N}$, et al. (2014) Effect of the glycemic index of the diet on weight loss, modulation of satiety, inflammation, and other metabolic risk factors: a randomized controlled trial. Am J Clin Nutr 100, $27-35$.

30. Rosén LA, Ostman EM \& Bjorck IM (2011) Postprandial glycemia, insulinemia, and satiety responses in healthy subjects after whole grain rye bread made from different rye varieties. J Agric Food Chem 59, 12149-12154.

31. Kong KL \& Hendrich S (2012) Glycemic index, insulinemic index, and satiety index of kefir. J Am Coll Nutr 31, 280-287.

32. Simmons AL, Miller CK, Clinton SK, et al. (2011) A comparison of satiety, glycemic index, and insulinemic index of wheat-derived soft pretzels with or without soy. Food Funct 2, 678-683.

33. Gonzalez-Anton C, Rico MC, Sanchez-Rodriguez E, et al. (2015) Glycemic responses, appetite ratings and gastrointestinal hormone responses of most common breads consumed in Spain. A randomized control trial in healthy humans. Nutrients $\mathbf{7}$, 4033-4053.

34. Ball SD, Keller KR, Moyer-Mileur LJ, et al. (2003) Prolongation of satiety after low versus moderately high glycemic index meals in obese adolescents. Pediatrics 111, 488-494. 